\title{
Gegenständliche Modellierung virtueller Informationswelten
}

\author{
Eva Hornecker und Kai Schäfer \\ Forschungszentrum Arbeit und Technik (artec), Universität Bremen \\ Veröffentlicht in: Software Ergonomie '99, U. Arend, E. Eberleh, \\ K. Pitschke (Hrsg.), Teubner, Stuttgart, 1999
}

\section{Zusammenfassung}

In diesem Beitrag stellen wir ein Konzept greifbarer, gegenständlicher Benutzungsschnittstellen vor. Es werden Übergänge zwischen dem Modellieren im Realen und dem Erstellen virtueller Modelle geschaffen. Synchron zum gegenständlichen Modell entsteht ein virtuelles Abbild, das für Simulation, Animation und für Hilfen genutzt werden kann. Intuitive, spielerische und vorbegriffliche Herangehensweisen werden auf diese Weise mit abstraktem, analytischem Vorgehen verbunden. Es werden Anwendungsbeispiele gegeben und ein Projekt näher erläutert, in dem Förderbandanlagen synchron im Gegenständlichen und Virtuellen modelliert werden. Dabei wird das Programmieren durch Vormachen eingesetzt: Durch Bewegen von Gegenständen durch das Modell werden Regeln für Steuerungen von Förderbändern generiert. Anschließend stellen wir dar, wie gegenständliche Modellumgebungen, die durch ein synchronisiertes Computermodell unterstützt werden, die Zusammenarbeit fördern, indem sie auf natürliche Weise einen gemeinsamen Interaktionsraum herstellen, dessen zentrale Eigenschaften erläutert werden.

\section{Summary}

In this text we present a concept of graspable computer-interfaces. It links modeling in physical reality with building virtual models. Synchronous to the graspable model a virtual twin-model is generated. The latter can be used for simulation, animation and giving help on the subject. Thus we connect intuitive, game-like and preconceptual approaches with abstract and analytic ways of thinking. We present some application areas, focusing on a project for the modeling of conveyor systems. We explain Programming by Demonstration: manually demonstrating the movement of objects through the scene we generate rules for programmable controls for the conveyor system. Then we focus on how working in computer supported concrete environments fosters group work and communication because it provides a shared workspace in a natural way.

\section{Einleitung}

Stellen Sie sich eine Fahrschule der Zukunft vor. Die Lernenden spielen zusammen Verkehrssituationen durch. Aber sie haben diese weder als Text noch als Zeichnung vor sich - sie spielen diese mit Hilfe von Spielzeugmodellen nach. Sie wählen eine Basisplatte mit dem Abbild einer Kreuzung, auf der einige Verkehrsschilder bereits plaziert sind, verändern diese evtl. durch Hinzufügen weiterer Schilder und setzen Modellautos, Fahrräder, Menschenfiguren auf Startpositionen am Rand der Platte. Jeder der Lernenden bewegt dann ein bis zwei Figuren über die Platte. Plötzlich leuchtet der Untergrund rund um zwei der Fahrzeugmodelle auf und aus einem Lautsprecher kommt eine Stimme: "Nicht eingehaltene Regel: 'Rechts hat Vorfahrt' sowie 'Fußgänger am Zebrastreifen hat Vorrecht'." Die Lernenden diskutieren die Situation, winken dann aber einen Fahrlehrer herbei. Da sie die gespielte Situation nicht mehr gänzlich aus dem Kopf rekonstruieren können, sehen sie sich gemeinsam die Aufzeichnung des Computers an, die in Form einer Animation gezeigt wird und analysieren diese. 
Dieses bewußt spielerisch gehaltene Beispiel soll hier die Möglichkeiten aufzeigen, die unsere grifforientierte, gegenständliche Benutzungsschnittstelle eröffnet. Leitidee unserer Arbeitsgruppe ist es, Übergänge zwischen dem Modellieren im Realen und dem Erstellen virtueller Modelle zu schaffen. Wie die Erfahrungen in mehreren Industriekooperationen zeigten, werden gegenständliche Modelle (z.B. von Fabrikanlagen und Hallenlayouts) nach wie vor aufgrund ihrer Anschaulichkeit und Kommunikationsförderlichkeit bevorzugt. Virtuelle Modelle im Computer sind jedoch für Animation, Simulation, Analyse und Archivierung notwendig. Daraus entstand das Ziel, die Modellarten zu koppeln und Übergänge zu schaffen.

Während der Modellierung im Realen wird synchron ein Modell im Rechner erstellt, vermittelt über moderne Interfacetechniken, die im folgenden noch erläutert werden. Das gegenständliche, greifbare Modell ist selber die Benutzungsschnittstelle. Die Modellierung mit realstofflichen Elementen und die technisch ungebrochene Kommunikation mit ihnen und über sie steht im Vordergrund [1] [2]. Der "Rechner im Rücken" soll unterstützendes Werkzeug und Medium für die Anwender sein. Bisher klafft eine Lücke zwischen den "Reality Techniken" Virtual Reality und Augmented Reality; der Bereich des Arbeitens mit den eigentlichen stofflichen Gegenständen wird unzureichend ausgefüllt. Da wir uns auf gegenständliche Modelle als Eingabemedium und Interaktionsraum konzentrieren und damit diese Lücke schließen, haben wir unser Konzept 1993 intern "Real Reality" genannt und den Begriff in [3] eingeführt.

Modellierung und Diskussion über Modelle sind für viele Lern- wie Arbeitsbereiche typische Situationen, in denen Real Reality Systeme eingesetzt werden können. Die gegenständliche Modellierung ermöglicht einen intuitiven Zugang, da sie an alltägliche Handlungsweisen anknüpft und nicht-sprachliche, vorbegriffliche Modellbildung erlaubt. Sie ist anschaulich und spricht das räumliche Denken an. Unsere Kopplung realer Modelle mit virtuellen verbindet das abstrahierende, distanzierende Vorgehen - das typischerweise von technischen Modellbildungssystemen unterstützt wird - mit dem intuitiven, spielerischen, sich einlassenden Vorgehen [1] und erleichtert die vielperspektivische, kreative Kommunikation. Real Reality Umgebungen ermöglichen und fördern so ein subjektivierendes Arbeitshandeln, wie es vom Arbeitswissenschaftler Fritz Böhle beschrieben wurde [4]. Unsere These besagt, daß ein hoher Grad an Stofflichkeit von Modellen positiven Einfluß auf Verständnis, Ideenreichtum und Kommunikation der Modellierenden hat.

\section{Modellieren mit Real Reality}

Wenn wir in einer Real Reality Umgebung modellieren, geschieht dieses nicht vor einem Computer, sondern mit alltäglichen Gegenständen oder mit Modellbausteinen. Lediglich zwei Voraussetzungen müssen für die stetige Aktualisierung des Computermodells erfüllt werden:

1. Die Geometrie und die Anfangsposition der Modellteile müssen im Computer als virtuelles Modell vorliegen.

2. Alle Modellierenden müssen bei ihrer Tätigkeit Datenhandschuhe tragen.

Nun können vorhandene Gegenstände beliebig bewegt werden, das Computermodell wird automatisch angepaßt. Zu jedem verwendeten Bauelement wird ein entsprechendes virtuelles Objekt erzeugt. Jeder Zustand des realen Modells hat ein kongruentes virtuelles Abbild im Rechner [2]. Die Paare aus virtuellem und realem Objekt heißen Complex Objects und können jeweils über unterschiedliche Eigenschaften verfügen. 
Wichtig ist, daß während der Modellierung die Modellobjekte selbst die Informationsträger sind. Das virtuelle Computermodell dient lediglich zu Analyse- und Dokumentationszwecken und bleibt während der Modellierung im Hintergrund. Für die Grifferkennung sind nur die Außenkonturen der Gegenstände relevant, so daß auf eine exakte Geometrie verzichtet werden kann. Neben den in Abb. 1 abgebildeten Bauklötzen verwenden wir häufig Fischertechnik, so daß sowohl im Gegenständlichen wie im Virtuellen unterschiedliche Abstraktionsstufen möglich sind.
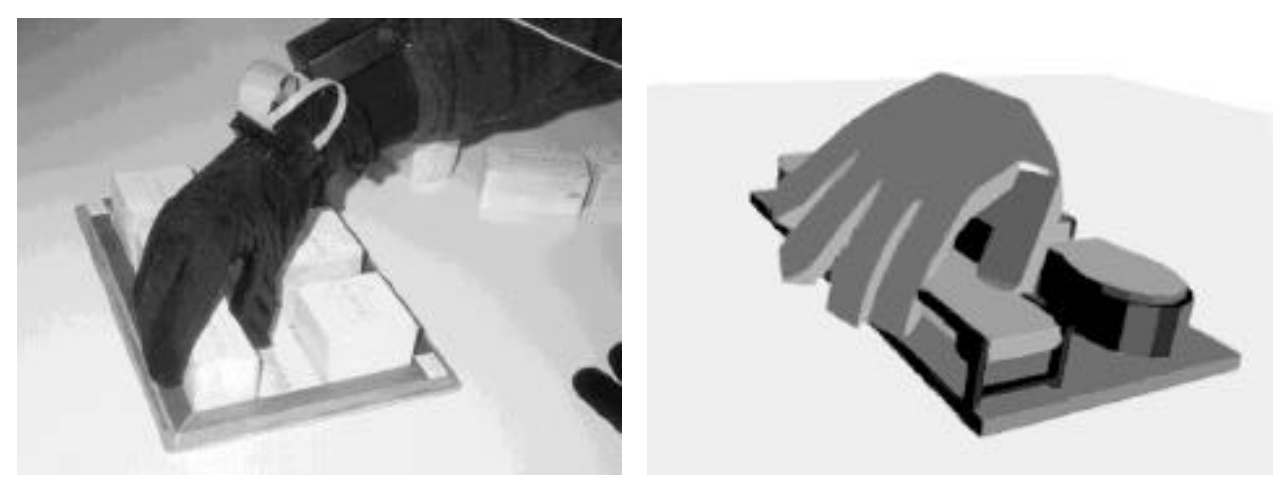

Abb. 1: Synchrone Modellierung im Realen und Virtuellen

Alle Veränderungen im Szenario gehen von der sensorisierten Hand aus. Mit Hilfe des Handschuhs werden Griffe erkannt. Die Position und Orientierung der Hand wird über ein elektromagnetisches Trackingsystem räumlich bestimmt. Entsprechen die Krümmungswerte am Handschuh einem Griffmuster, verknüpft die Real Object Manipulator Software (ROMAN) ein reales mit dem zugehörigen virtuellen Objekt und bewegt diese jetzt synchron, bis der Griff durch Verlassen des Griffmusters gelöst wird (Abb. 1) [3]. Die Architektur des ROMANs erlaubt die gleichzeitige Verwendung mehrerer Handschuhe.

Wenn wir mit Gegenständen handeln und modellieren, hat dieses für uns eine Bedeutung. Das ist für die Real Reality Software nicht der Fall. Diese behandelt alle Objekte gleich und verarbeitet lediglich die für die Handhabung relevanten Attribute Position, Orientierung und Geometrie. Aus diesem Grund läßt sich das Real Reality Konzept für jeden beliebigen Kontext universell einsetzen. Die unteren vier Ebenen in Abb. 2 stellen diesen Kern von Real Reality dar. Den Bezug zu den Anwendungs- und Modellkontexten stellen nachgeschaltete Softwarekomponenten durch die weitere Verarbeitung der Modelldaten her.

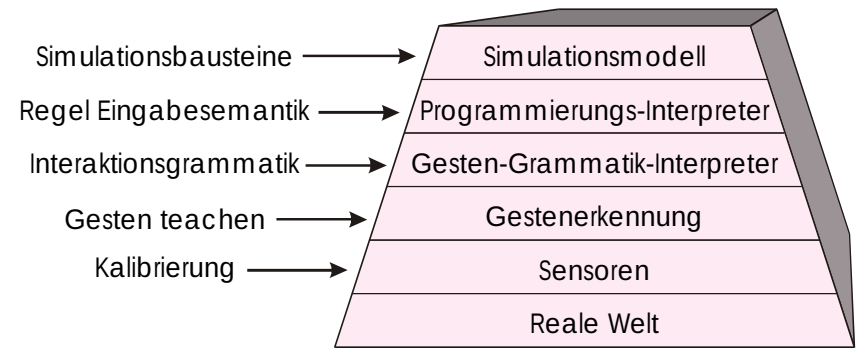

Abb. 2: Automatische Abstraktion der realen Welt

Der ROMAN verwaltet für die kontextspezifische Verarbeitung beliebige benutzerdefinierte Attribute, die mit den Objekten verknüpft sind. Die Modellierung erhält hierdurch eine se- 
mantische Bedeutung, die sich für festgelegte Anwendungsfälle durch spezifische Software automatisch verarbeiten läßt. Beim Modellieren werden nicht nur die aktuellen Positionen der Objekte gespeichert, sondern auch die Bahnen, auf denen sie bewegt wurden. Dieses ermöglicht es, dynamisches Verhalten durch Vormachen am gegenständlichen Modell zu programmieren, es zu interpretieren und zu simulieren. Die durchgeführten Bewegungen und Aktionen werden dabei zu abstrakten Steuerprogrammen für Simulatoren und Maschinen verarbeitet. Über einen mehrstufigen Abstraktionsprozeß (Abb. 2, die beiden oberen Ebenen), können aus Modellen in der realen Welt simulierte und animierte Welten im Computer geschaffen werden.

\section{Anwendungsbereiche}

Der erste Anwendungsbereich, in dem Real Reality eingesetzt wurde, ist die Modellierung von Förderbandanlagen für die Produktionstechnik [8]. Das Verhalten der Anlage kann mittels manuellen Programmierens durch Vormachen spezifiziert und in einem Simulator evaluiert werden. Dies wird im folgenden genauer dargestellt. In einem anderen Projekt wird eine Lernumgebung für den Pneumatikunterricht an beruflichen Schulen entwickelt [6]. Parallel zum Aufbau einer Schaltung mit gegenständlichen Symbolen oder aus echten Pneumatikbauteilen entsteht simultan in einem Pneumatiksimulator ein Abbild der Schaltung, das simuliert und evaluiert werden kann. Der Simulator kann durch Gesten gesteuert werden und liefert z.B. Hilfe zu einem Bauelement oder startet und stoppt den Simulationslauf. Die Lernumgebung soll fließende Übergänge zwischen dem erfahrungsorientierten Lernen im Realen und dem Umgang mit Symbolen in Schaltplänen und Simulatoren ermöglichen und handlungsorientierten Unterricht unterstützen.

In einem laufenden Projekt wird die Freiformmodellierung im Sinne einer Skizzierungsmethode untersucht. Die ModelliererIn verformt mit ihren sensorisierten Händen eine Modelliermasse. Alle Bewegungen werden aufgezeichnet, um einen dreidimensionalen Freiformkörper zu generieren. Gesten und synchrone Spracheingaben spezifizieren das Endziel weiter.

Eine mögliche weitere Anwendung wäre eine kombiniert real-virtuelle Plantafel mit in Größe, Farbe und Form unterscheidbaren Zeit- und Auftragsbausteinen. Die reale Plantafel bietet aufgrund ihrer Größe und Physikalität einen besseren Überblick und einen intuitiven Umgang. Der virtuelle Teil kann zur Konsistenzprüfung und zur Dokumentation eingesetzt werden. In der Einleitung wurde bereits das Szenario eines Lernsystems für Fahrschulen dargestellt, in dem das gegenständliche Spiel von Verkehrssituationen vom Computer auf die Einhaltung der Verkehrsregeln geprüft wird. In einem umgekehrten Ansatz sind Real Reality Systeme zur Verkehrs- und Stadtplanung denkbar, in denen Straßen gegenständlich modelliert werden und der Verkehrsfluß manuell vorgemacht wird. Architekten, Stadtplaner und Anwohner können anhand des allen verständlichen Modells diskutieren und daran Änderungen vornehmen. Das gegenständliche Modell wird von der Anwendung in Planskizzen etc. umgewandelt; das virtuelle Modell läßt sich beispielsweise zur Simulation des Verkehrsablaufes nutzen.

\section{Erstellen von Steuerprogrammen für industrielle Automatisie- rungseinrichtungen durch Vormachen}

Vergleichende Studien zur Simulation und Modellierung von Hallenlayouts mit Förderbändern, Verzweigungen, Bearbeitungsstationen haben gezeigt, daß verschiedene Resultate von 
verschiedenen Personen mit verschiedenen Simulatoren erzielt wurden [7]. Zum einen lag das an Unklarheiten in der Aufgabenstellung, die von den Modellierenden aufgrund mangelnder Anschaulichkeit nicht erkannt wurden und zum anderen an der unterschiedlichen Implementierung des Verhaltens der Simulationsbausteine, die zu unkritisch ins Modell eingesetzt wurden. Wenn das Verhalten der Anlage an einem Modell mit kleinen Paletten vorgemacht wird, ist nicht zu erwarten, daß solche Fehler unbemerkt bleiben. Im Projekt RUGAMS ${ }^{1}$ wird deshalb ein solches Problem gegenständlich modelliert, um zu zeigen, daß Hallenlayout und Anlagensteuerung mit Real Reality geplant und simuliert werden können [8].

Bereits in der Entwurfsphase wird eine ereignisorientierte Computersimulation zukünftiger Fertigungsabläufe ermöglicht. Jeder Baustein ist hierfür mit einem charakteristischen Vorgabeverhalten ausgestattet. Die topologische Analyse des virtuellen Computermodells verknüpft die einzelnen Simulationsbausteine zu einem lauffähigen Gesamtmodell. In darauf folgenden Phasen kann das Verhalten des Modells durch Vormachen weiter beeinflußt werden (Abb. 3).

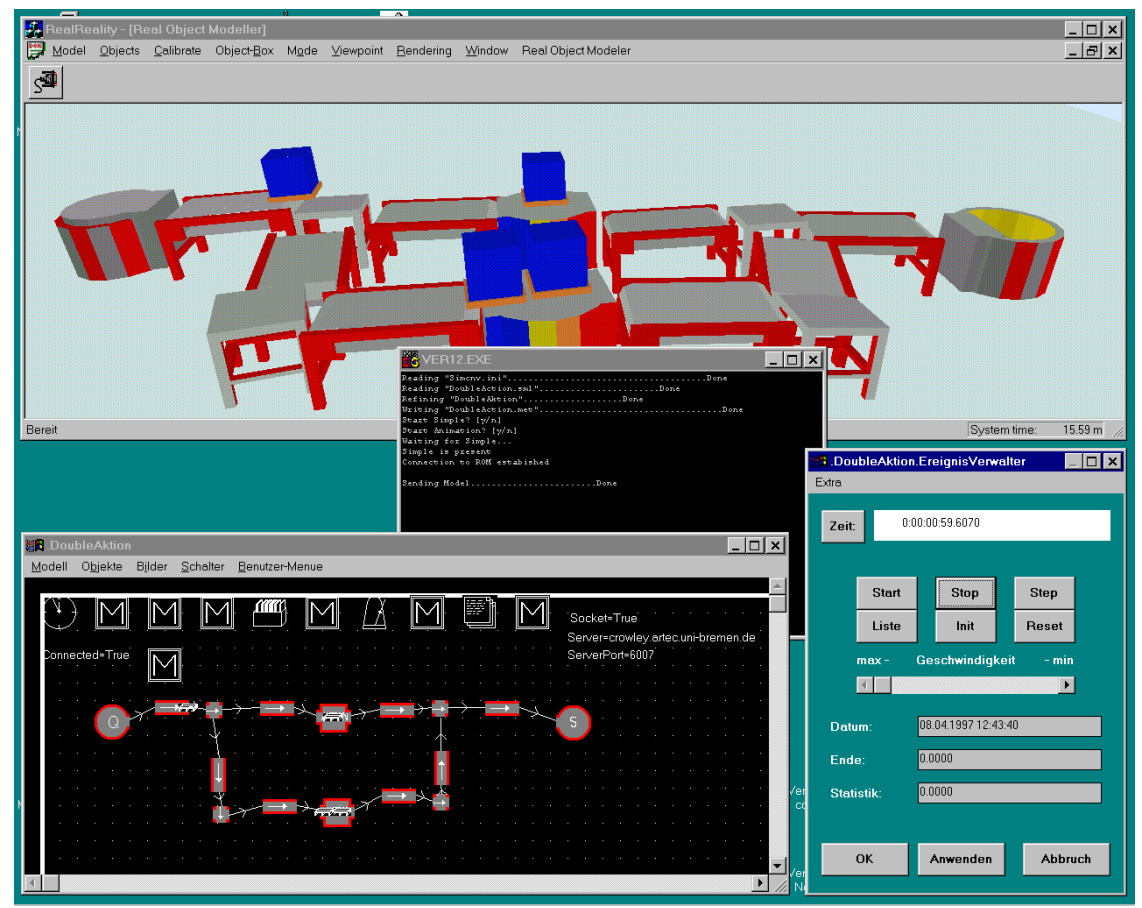

Abb. 3: Visualisierung von Modelldynamik am VR Modell

Beim Vormachen der Route von Werkstücken durch das System wird die Reihenfolge überfahrener Positionen aufgezeichnet. Diese Sensepathes werden zusammen mit der Topologie und Informationen über die Förderbausteine als Kontextwissen für eine Regelerkennung genutzt. Wird ein Verzweigungspunkt überfahren (Abb. 4 links), wird eine Entscheidungsregel generiert und als Attribut der Verzweigung gespeichert. Der Bearbeitungszustand des Werkstücks, der durch die Farbe des Klotzes repräsentiert ist, wird in die Verzweigungsregel einbezogen. Wenn das vorgegebene Verteilungsverhalten an der Verzweigung z.B. zufällig oder alternierend war, wird an der Verzweigung jetzt auf alle Paletten mit gleicher farblicher

\footnotetext{
1 Rechnergestützte Übergänge zwischen gegenständlichen und abstrakten Modellen produktionstechnischer Systeme (DFG)
} 
Kennzeichnung das vorgemachte Verhalten angewandt. Alle anderen Verzweigungsentscheidungen werden weiterhin nach dem Vorgabeverhalten des Förderbausteins behandelt [9].

Zusätzlich können Verzweigungen in Abhängigkeit von Maschinen- und Pufferbelegungen spezifiziert werden. Reale Anlagen sind hierfür mit Sensoren ausgestattet. Entscheidungsrelevante Sensorpositionen werden vor dem Überfahren des Verzweigungsknotens in ihrem aktuellen Belegungszustand mit Token gekennzeichnet (Abb. 4 rechts). Neben dem Zustand des Materialflußelements hängt die Verzweigung jetzt von der Verfügbarkeit einzelner Ressourcen $a b$. Werden verschiedene widersprüchliche Verzweigungsregeln für eine Situation vorgemacht, werden diese alternierend angewendet. Das vorgemachte Verhalten wird von einer wissensbasierten Regelanalyse verarbeitet, die das Verhalten in einer Programmiersprache für Anlagensteuerungen (AWL für SPS) ausgibt.
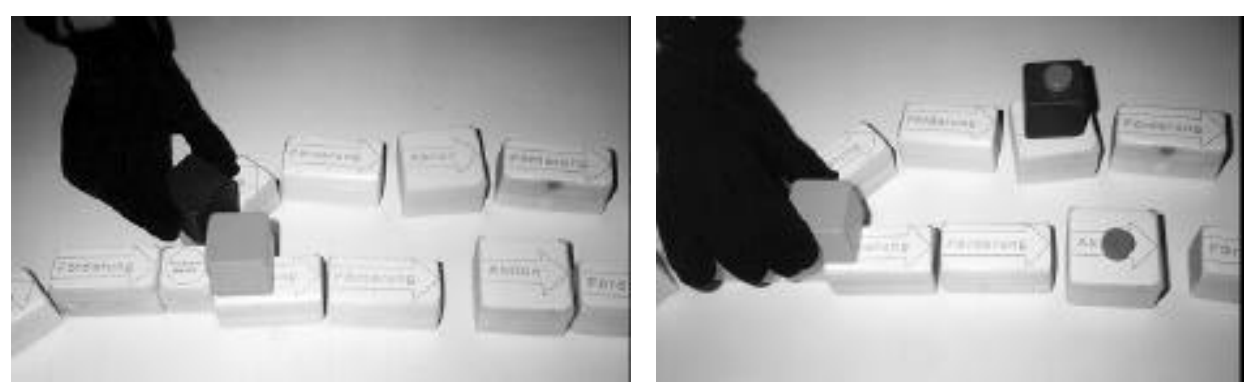

Abb. 4: Vormachen einer Verzweigungsregel

Aus der Modelltopologie, dem Vorgabeverhalten für die Bausteintypen und dem vorgemachten Verhaltensregeln läßt sich automatisch das Simulationsmodell aufbauen. Zur Zeit existiert eine Schnittstelle zum industriellen Materialflußsimulator SIMPLE++. Wenn dieses Modell in den Simulator geladen und ausgeführt wird, kann das von der geplanten Anlage zu erwartende Verhalten auf dem Bildschirm beobachtet und beurteilt werden. Da die Animation des Simulators auf dem Bidschirm sehr abstrakt und damit nicht für jeden verständlich ist (Abb. 3, links unten), werden die Veränderungen auf das dreidimensionale virtuelle Modell übertragen und dort visualisiert. Da der Wechsel vom Arbeitstisch zum Computer einen Medienbruch darstellt, projizieren wir die Bildschirmausgabe häufig mit einem Videoprojektor (Beamer) auf den Tisch und in das gegenständliche Modell hinein (Abb. 5). In diesem Sinne verwenden wir Augmented Reality Techniken, um die greifbare Repräsentation durch die dynamische Simulationsvisualisierung zu erweitern. Die große Ausgabefläche erleichtert die Diskussion in der Gruppe und erhöht die Beteiligung spürbar. Auf dieser Visualisierung aufbauend kann das Modell auf dem Tisch verändert und neu programmiert werden, bis es den Anforderungen der Akteure entspricht. 


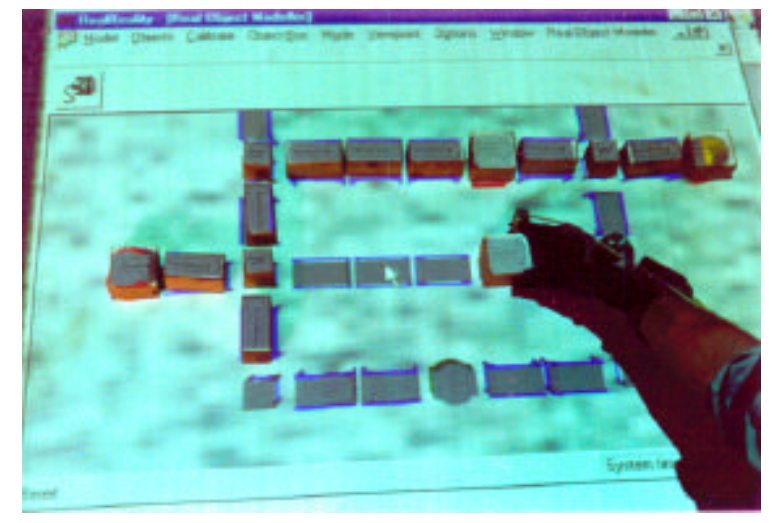

Abb. 5: Statische und dynamische Informationen werden ins Modell eingeblendet

Der besondere Vorzug des Programmierens durch Vormachen am gegenständlichen Modell ist die Intuitivität beim Umgang mit den Objekten und deren Verhalten, die es allen Beteiligten erlaubt, sich aktiv in den Planungsablauf einzubringen. Gute Modelle sind jene, die passende Ideen von allen Modellierenden berücksichtigen und die von allen Beteiligten verstanden und akzeptiert sind. Anders als bei klassischen Simulatoren ist eine kooperative Modellierung in der Gruppe möglich, die es unterschiedlichsten Qualifikationsstufen erlaubt, sich auszudrücken, aktiv zu werden und das Ergebnis zu verstehen.

\section{Real Reality als kooperationsunterstützendes Arbeitsmedium}

Die verbreiteten computergestützten Modellierungs- und Simulationswerkzeuge erfordern es, am Monitor zu arbeiten, auch wenn alle Interaktionspartner anwesend sind. Die Sicht auf den Bildschirm beschränkt die Gruppengröße; Tastatur und Maus restringieren die aktive Kontrolle. Real Reality ermöglicht dagegen die Arbeit in einem gemeinsamen Interaktionsraum und stellt dabei Computerunterstützung zur Verfügung, wie in Abb. 6 zu sehen.

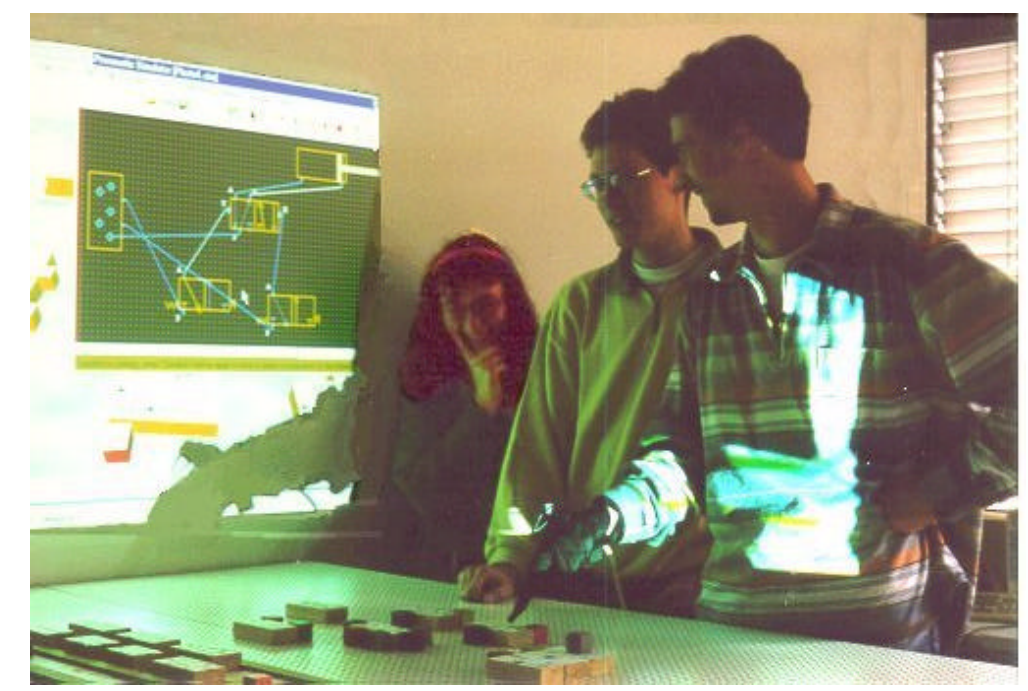

Abb. 6: Gemeinsame Arbeit am Modelliertisch, im Hintergrund die Projektion

Kooperatives Lernen und Arbeiten wird durch die Arbeit in einem solchen gemeinsam geteilten Interaktionsraum erleichtert. Die Schwierigkeiten, die durch das Fehlen sozialer Hin- 
weisreize und Präsenz (nonverbale Kommunikation), fehlende Gruppenkoordination und "awareness" (Wahrnehmung der Handlungen und Intentionen der Interaktionspartner) entstehen, werden in vielen Studien zur computerunterstützten Kommunikation deutlich. Videokonferenzsysteme erhöhen den Grad der sozialen Präsenz, ermöglichen in der Regel jedoch nicht das gesamte Spektrum nonverbaler Kommunikation, da die Teilnehmer(bilder) in keinem räumlichen Bezug zueinander stehen, wodurch z.B. kein Blickkontakt entsteht. Als effektiver hat sich die Sichtbarkeit der gemeinsam bearbeiteten bzw. diskutierten Objekte erwiesen, die als gemeinsame Referenz den Konversationsinhalt koordinieren (video as data) [10]. Daher wird viel Aufwand in die Schaffung von "shared workspace" investiert; gemeinsame Arbeitsbereiche, in denen z.B. die Stelle, an der eine Kollegin gerade arbeitet, visuell markiert ist oder ihr Sichtbarkeitsbereich durch einen Kasten repräsentiert wird. [11]

Mehrere Studien zeigen die Vorteile von realen gemeinsamen Interaktionsräumen auf und beschreiben ihre Merkmale. Rauterberg [12] und Suzuki [13] argumentieren, daß durch einen solchen "shared social space" Kommunikation und gemeinsam geteiltes Verständnis gefördert werden. In ihm ist das gesamte Spektrum verbaler und nonverbaler Kommunikation offen. Seine Besonderheiten [14] bestehen darin, daß er drei wichtige Kommunikationsaspekte unterstützt: Sichtbarkeit, Hörbarkeit und soziale Nähe. Dies wird von Böhme [15, S. 177, 211] gestützt, der Kommunikation als Ausübung und Vermischung von Präsenz beschreibt. Dabei sei die leibliche Anwesenheit wichtig, denn der Mensch strahle eine spürbare Atmosphäre aus, die sich durch ihre Konkretheit und Lebendigkeit auszeichne.

Die Gegenständlichkeit - als Möglichkeit zum Greifen und Zeigen - erleichtert die Verständigung, insbesondere wenn noch keine gemeinsamen Begriffe existieren oder die Fachtermini fremd sind [13] [16]. Dies gilt sowohl für heterogene Entwurfsgruppen [17], wie für Lernende, die eine Fachsprache erst unvollkommen beherrschen. Die gegenständlichen Symbole sind für alle sichtbar und greifbar, können von Hand zu Hand weitergereicht werden. Dinge werden zu Werkzeugen des Denkens und Kommunizierens, wie D. Norman am Beispiel des Vorspielens eines Autounfalls mit zufällig ergriffenen Gegenständen erläutert: "We can make marks or symbols that represent something else and then do our reasoning by using these marks. (... They) help the mind keep track of complex events. (... This) is also a tool for social communication. (...) The tabletop becomes a shared workspace with shared representations of the event." [18]. Es gibt zudem Hinweise, daß die manuelle Aktivität zu mehr Reflektion vor der Ausführung einer Handlung führt, die Sichtbarkeit und (scheinbare) Endgültigkeit die Verbindlichkeit erhöht [19]. Daher interessiert uns, wie Menschen in ihren Denk- und Kommunikationsprozessen mit Gegenständen interagieren und wie die Gegenstände sie darin beeinflussen. Bisher scheinen nur vereinzelte Studien ähnliche Fragen zu untersuchen (z.B. [20]).

Der gemeinsame Interaktionsraum ist im Real Reality Ansatz durch die Gegenstände und den Modelltisch selbst gegeben und muß nicht erst durch elektronische Hilfsmittel geschaffen werden. Da mehrere Handschuhe gleichzeitig verwendet werden können, ist die parallele Arbeit mehrerer Personen am Modell möglich. Dies bezieht sich auch auf das Programmieren durch Vormachen, das ebenfalls ein kooperativer, diskursiver Prozeß sein kann. Mehrere Personen können alternierend oder sogar gleichzeitig Regeln vormachen, da alle Bewegungen der Objekte aufgezeichnet werden. Denkbar wären spezielle Regelgenerierungsmodi, in denen vorgemachte Regeln je nach Benutzer anders zugeordnet werden oder parallel vorgemachte Regeln gemeinsam interpretiert werden. 
Eine offene Frage besteht darin, wie bei einer interaktiven Steuerung eines Anwendungsprogrammes (z.B. des Pneumatiksimulators) vom Arbeitstisch aus gleichzeitige oder interferierende Befehle an den Computer behandelt werden. Bei einer visuellen Ausgabe ergeben sich Fragen der Zeitlichkeit, der Anordnung, der Zuordnung, sowie Platzeinschränkungen. Je nach Gestensprache sind weiterhin widersprüchliche gleichzeitige Befehle möglich. Ein Sperren von Aktionsmöglichkeiten, wie in Computersystemen, ist im Realen schwer möglich. Für die weitere Arbeit am Real Reality-Konzept ergeben sich interessante konzeptionelle Fragen. Wie lassen sich die hochgradig parallelen, nur bedingt synchronisierten, zum Teil widersprüchlichen und sozial ausgehandelten Interaktionen adäquat im Anwendungsprogramm spiegeln und verarbeiten, ohne den Benutzern ein Verhaltenskorsett und inadäquate Kommunikationsmodelle aufzuerlegen? Inwieweit kann auf aufwendige Lösungen verzichtet werden, weil die Benutzer in der Lage sind, ihr Arbeiten und die Reihenfolge von Aktionen unter sich auszuhandeln und abzustimmen?

\section{Zusammenfassung und Einordnung}

Unsere Forschungsperspektiven beinhalten die Kombination mit Telekooperation, die Erweiterung des Programmierens durch Vormachen, die Ergänzung durch Visualisierungstechniken der Augmented Reality, Konzepte zur Synchronisierung paralleler Aktionen im Anwendungsprogramm, die Erkundung von Merkmalen geeigneter Anwendungsbereiche, Eingabe-

semantiken und Interaktionsgrammatiken für Gestensprachen. Schwierigkeiten bereitet die uns verfügbare Hardware, die keine optimale Grifferkennung und Positionsbestimmung ermöglicht. Das elektromagnetische Trackingsystem wird zudem durch metallische Objekte beeinflußt und läßt deren Verwendung nicht zu.

Unser Ansatz hat mit Augmented Reality das Ziel gemeinsam, den Menschen in seiner alltäglichen Umwelt integriert zu lassen und einen intuitiven Umgang mit Benutzungsschnittstellen zu ermöglichen (siehe z.B. [21]). Verschiedene Augmented Reality Projekte befassen sich mit kooperativen Arbeitsumgebungen für gleichzeitig anwesende Personen, häufig unter Einblendung virtueller Gegenstände in halbdurchlässige Head-Mounted-Displays (siehe z.B. [22]). Auch Konzepte wie die elektronischen WhiteBoards oder "Roomware" [23] integrieren aktives und reaktives Verhalten in Gegenstände bzw. ganze Räume, um kreative kooperative Arbeit zu unterstützen.

Unser Ansatz läßt sich jedoch eher mit den sog. "graspable user interfaces" vergleichen. Ein wichtiges Prinzip von Real Reality ist es, alle Modellobjekte greifbar zu machen, dreidimensional und haptisch erfaßbar, nicht nur die Interaktionswerkzeuge (etwa in Form greifbarer Zeiger und Manipulationswerkzeuge). Dies unterscheidet Real Reality von Projekten wie Build-It [12] oder "tangible bits" des MIT [24]. In der Verbindung von Greifbarkeit aller Modellobjekte und Simulationsvisualisierung kommt das "Illuminating Light" Projekt des MIT [25] unserem Vorgehen am nächsten. Von diesem und den Labilbaukästen wie Triangles [26] oder AlgoBlocks [13] unterscheidet sich Real Reality wiederum durch die Sensorisierung der Hand (anstatt die physikalischen Objekte zu sensorisieren). Gesten werden durch Vormachen definiert, sie wirken sich zunächst auf alle Dinge gleich aus. Sie können aber auch ohne Objektbezug interpretiert werden. Durch die Sensorisierung der Hand wird zudem die Auswahl von Objekten vereinfacht, da diese nicht sensorisiert werden müssen. 
Wir danken unseren Kollegen bei artec, die am Entstehen des Konzeptes und der beschriebenen Gedanken maßgeblich mitgewirkt haben: Prof. Willi Bruns, Volker Brauer, Bernd Robben, Ingrid Rügge, Dieter Müller, Achim Heimbucher, sowie unseren studentischen Mitarbeitern.

\section{Literaturangaben}

[1] W. Bruns: Sinnlichkeit in der Technikgestaltung und Technikhandhabung - Ein konstruktiver Ansatz. In: C. Schachtner (Hg.): Technik und Subjektivität. 1997: suhrkamp taschenbuch wissenschaft.

[2] F. W. Bruns: Zur Rückgewinnung von Sinnlichkeit - Eine neue Form des Umgangs mit Rechnern. CH, 1993: Technische Rundschau Heft 29/30, 85. Jahrgang.

[3] F. W. Bruns, V. Brauer: Bridging the Gap between Real and Virtual Modeling - A new Approach to Human-Computer-Interaction. IFIP-Workshop "Virtual Prototyping", Texas 1996 (Auch als artec Paper Nr. 46, Universität Bremen).

[4] F. Böhle, B. Milkau: Vom Handrad zum Bildschirm - eine Untersuchung zur sinnlichen Erfahrung im Arbeitsprozeß. 1988: Campus Verlag.

[5] K. Schäfer, V. Brauer, F. W. Bruns: A new Approach to Human-Computer Interaction; Synchronous Modelling in Real and Virtual Spaces. DIS ‘97. 1997: ACM.

[6] B. Robben, E. Hornecker: Gegenständliche Modelle mit dem Datenhandschuh begreifen - Eine Lernumgebung für den Technikunterricht. In: V. Claus (Hg.): Informatik und Ausbildung '98. 1998: Springer.

[7] J. Krauth: Comparison 2, Flexible Assembly System. EUROSIM: Simulation News 2, May 1992.

[8] V. Brauer, W. Bruns, K. Schäfer: Rechnergestützte Übergänge zwischen gegenständlichen und abstrakten Modellen produktionstechnischer Systeme, Erster und zweiter Zwischenbericht zum DFG Forschungsprojekt RUGAMS. Bremen, 1998: artec Arbeitspapier 56.

[9] K. Schäfer: Real Reality - Simulationsunterstützung durch gegenständliche Modelle. Tagungsband Simulation und Visualisierung '98. Magdeburg, 1998: SCS.

[10] S. Whittaker: Rethinking video as a technology for interpersonal communications: theory and design implications. In: International Journal of Human-Computer-Studies (1995) 42

[11] R.B. Smith: What You See Is What I Think You See. In: Conference on Computer Supported Collaborative Learning 1992, Vol 21 \#3, 192: ACM.

[12] M. Rauterberg, P. Steiger. Pattern Recognition as a Key Technology for the Next Generation of User Interfaces. In: 1996 IEEE International Conference on Systems, Man and Cybernetics - Information Intelligence and Systems, Vol 4 of 4. 1996: IEEE.

[13] H. Suzuki, H. Kato. Interaction-Level Support for Collaborative Learning: AlgoBlock - An Open Programming Language. In: Conference on Computer Supported Cooperative Learning '95. 1995.

[14] M. Rauterberg, M. Sperisen, M. Däwyler (1995): From Competition to Collaboration through a Shared Social Space. In: B. Blumental, J. Govnostaev, C. Unger. (eds.): East-West International Conference on Human Computer Interaction - EWHCI '95 (Vol II), Moskau: International Centre for Scientific \& Technical Information, 1995.

[15] G. Böhme: Einführung in die Philosophie - Weltweisheit Lebensform Wissenschaft. 2. Auflage. 1997: suhrkamp taschenbuch wissenschaft.

[16] E. Arias, H. Eden, G. Fischer. Enhancing Communication, Facilitating Shared Understanding, and Creating Better Artifacts by Integrating Physical and Computational Media for Design. In: DIS '97. 1997: ACM.

[17] J. Scheel, K. Hacker, K Henning: Fabrikorganisation neu beGreifen. Köln , 1994: TÜV Rheinland.

[18] D. A. Norman: Things that Make Us Smart - Defending Human Attributes in the Age of the Machine. 1994: Addison Wesley.

[19] S. Whittaker, H. Schwarz: Back to the Future: Pen and Paper Technology Supports Complex Group Coordination. In: CHI '95 Mosaic of Creativity 1995: ACM.

[20] I. Neilson, J. Lee: Conversations with graphics: implications for the design of natural language/graphics interfaces. In: International Journal of Human-Computer-Studies (1994) 40.

[21] P. Wellner, W. Mackay, R. Gold. Computer-Augmented Environments - Back to the Real World. In: Communications of the ACM, 36/7. 1993: ACM.

[22] Z. Szalavári, M. Gervautz. Interaktion mit virtuellen Informationen in realen Umgebungen - das "Personal Interaction Panel”. In: I. Rügge, B. Robben, E. Hornecker, W. Bruns (Hg.): Arbeiten und Begreifen: Neue Mensch-Computer-Schnittstellen. 1998: Lit-Verlag. 
[23] N. A. Streitz, J. Geißler, T. Holmer. Roomware for Cooperative Buildings: Integrated Design of Architectural Spaces and Information Spaces. In: N. A. Streitz, J. Konomi, H.-J. Burkhardt (Edt.): Cooperative Buildings. Proceedings of CoBuild '98. 1998: Springer.

[24] H. Ishii, B. Ullmer. Tangible Bits: Towards Seamless Interfaces between People, Bits and Atoms. In: CHI 97. 1997: ACM.

[25] J. Underkoffler, H. Ishii. Illuminating Light: An Optical Design Tool with a Luminous-Tangible Interface. In: CHI '98. 1998: ACM.

[26] M. G. Gorbet, M. Orth, H. Ishii. Triangles: Tangible Interface for Manipulation and Exploration of Digital Information Topography. In: CHI '98. 1998: ACM.

\section{Adressen der Autoren}

Eva Hornecker, Kai Schäfer

Universität Bremen

Forschungszentrum Arbeit und Technik (artec)

Enrique-Schmidt-Straße (SFG), Postfach 330440

D-28334 Bremen

Email: eva@artec.uni-bremen.de, schaefer@artec.uni-bremen.de 